\title{
Assessing lung transplantation ischemia-reperfusion injury by microcomputed tomography and ultrashort echo-time magnetic resonance imaging in a mouse model
}

\author{
Wurnig, Moritz C ; Tsushima, Yukio ; Weiger, Markus ; Jungraithmayr, Wolfgang ; Boss, Andreas
}

\begin{abstract}
PURPOSE: Ischemia-reperfusion injury (I/R) is a common early complication after lung transplantation. The purpose of this study was to compare ultrashort echo-time (UTE) sequences in magnetic resonance imaging (MRI) with a microcomputed tomography (micro-CT) reference standard for detection of $\mathrm{I} / \mathrm{R}$ injury in a lung transplantation mouse model. MATERIALS AND METHODS: Six mice (C57BL/6) underwent orthotopic lung transplantation using donor grafts that were exposed to 6-hour cold ischemia. Imaging was performed within 24 hours after the transplantation with high-resolution micro-CT (tube voltage, $50 \mathrm{kV}$; current, $500 \mathrm{~mA}$; aluminum filter, $0.5 \mathrm{~mm}$; voxel size, $35 \times 35 \times 35 \mathrm{~m}$ ) and small-animal MRI at $4.7 \mathrm{~T}$ with a linearly polarized whole-body mouse coil. The imaging protocol comprised radial 3-dimensional UTE sequences with different echo times (repetition time, 8 milliseconds; echo time, $50 / 75 / 100 / 500 / 1500 / 3000 / 4000 / 5000 \mathrm{~s}$; voxel size, $350 \times 350 \times 350 \mathrm{~m})$. Images were assessed visually and through calculation of contrast-to-noise ratio (CNR) values. Calculated S0 values and $\mathrm{T}_{2}^{*}$ transverse relaxation times (MRI) of lung parenchyma were compared with Hounsfield unit (HU) density in micro-CT images. Receiver operating characteristic curves and area under the curve values were calculated for comparison of diagnostic power. All samples underwent a histologic examination. RESULTS: The results of both UTE MRI and micro-CT showed an excellent depiction of pulmonary infiltration due to I/R injury, with MRI exhibiting a significantly higher CNR (mean [SD] CNR MRI, 19.7 [8.0]; mean [SD] CNR micro-CT, 10.3 [2.5]; $\mathrm{P}<0.001$ ). Measured parametrical values were as follows: mean (SD) HU, -416 (120); mean (SD) S0 value, 1655 (440); mean (SD) T2*, 895 (870) s for the non-transplanted right lung and mean (SD) HU, 29 (35); mean (SD) S0 value, 2310 (300); and mean (SD) $\mathrm{T} 2 *, 4550$ (3230) s for the transplanted left lung. Slight infiltration could be better discriminated with micro-CT, whereas, in strong infiltration, a better contrast was provided by UTE MRI. The area under the curve values resulting from the receiver operating characteristic curve analysis were 0.99 for HU density, 0.89 for S0, 0.96 for T2*, and 0.98 for the combination of S0 and T2*. CONCLUSIONS: Results show that MRI of the lung has a similar diagnostic power compared with that of micro-CT regarding the detection of $\mathrm{I} / \mathrm{R}$ injury after experimental lung transplantation. Both modalities provide complementary information in the assessment of dense and slight infiltration in the early phase after lung transplantation. Therefore, UTE MRI seems to be a promising addition to computed tomographic imaging in the assessment of I/R injury after lung transplantation.
\end{abstract}

DOI: https://doi.org/10.1097/RLI.0b013e3182a53111

Posted at the Zurich Open Repository and Archive, University of Zurich

ZORA URL: https://doi.org/10.5167/uzh-81311

Journal Article

Published Version 
Originally published at:

Wurnig, Moritz C; Tsushima, Yukio; Weiger, Markus; Jungraithmayr, Wolfgang; Boss, Andreas (2014). Assessing lung transplantation ischemia-reperfusion injury by microcomputed tomography and ultrashort echo-time magnetic resonance imaging in a mouse model. Investigative Radiology, 49(1):23-28.

DOI: https://doi.org/10.1097/RLI.0b013e3182a53111 


\title{
Assessing Lung Transplantation Ischemia-Reperfusion Injury by Microcomputed Tomography and Ultrashort Echo-Time Magnetic Resonance Imaging in a Mouse Model
}

\author{
Moritz C. Wurnig, MD, MSc, * Yukio Tsushima, MD, † Markus Weiger, PhD, $\neq$ \\ Wolfgang Jungraithmayr, $M D, P h D, \dagger$ and Andreas Boss, $M D, P h D^{*}$
}

\begin{abstract}
Purpose: Ischemia-reperfusion injury (I/R) is a common early complication after lung transplantation. The purpose of this study was to compare ultrashort echo-time (UTE) sequences in magnetic resonance imaging (MRI) with a microcomputed tomography (micro-CT) reference standard for detection of $\mathrm{I} / \mathrm{R}$ injury in a lung transplantation mouse model.
\end{abstract}

Materials and Methods: Six mice (C57BL/6) underwent orthotopic lung transplantation using donor grafts that were exposed to 6-hour cold ischemia. Imaging was performed within 24 hours after the transplantation with highresolution micro-CT (tube voltage, $50 \mathrm{kV}$; current, $500 \mathrm{~mA}$; aluminum filter, $0.5 \mathrm{~mm}$; voxel size, $35 \times 35 \times 35 \mu^{3}$ ) and small-animal MRI at $4.7 \mathrm{~T}$ with a linearly polarized whole-body mouse coil. The imaging protocol comprised radial 3-dimensional UTE sequences with different echo times (repetition time, 8 milliseconds; echo time, 50/75/100/500/1500/3000/4000/ $5000 \mu \mathrm{s}$; voxel size, $350 \times 350 \times 350 \mu^{3}$ ). Images were assessed visually and through calculation of contrast-to-noise ratio (CNR) values. Calculated $S_{0}$ values and $\mathrm{T} 2 *$ transverse relaxation times (MRI) of lung parenchyma were compared with Hounsfield unit (HU) density in micro-CT images. Receiver operating characteristic curves and area under the curve values were calculated for comparison of diagnostic power. All samples underwent a histologic examination.

Results: The results of both UTE MRI and micro-CT showed an excellent depiction of pulmonary infiltration due to I/R injury, with MRI exhibiting a significantly higher CNR (mean [SD] CNR MRI, 19.7 [8.0]; mean [SD] CNR micro-CT, 10.3 [2.5]; $P<0.001)$. Measured parametrical values were as follows: mean (SD) HU, -416 (120); mean (SD) $\mathrm{S}_{0}$ value, 1655 (440); mean (SD) T2*, $895(870) \mu \mathrm{s}$ for the non-transplanted right lung and mean (SD) HU, 29 (35); mean (SD) $\mathrm{S}_{0}$ value, 2310 (300); and mean (SD) T2*, 4550 (3230) $\mu \mathrm{s}$ for the transplanted left lung. Slight infiltration could be better discriminated with micro-CT, whereas, in strong infiltration, a better contrast was provided by UTE MRI. The area under the curve values resulting from the receiver operating characteristic curve analysis were 0.99 for $\mathrm{HU}$ density, 0.89 for $\mathrm{S}_{0}, 0.96$ for $\mathrm{T} 2 *$, and 0.98 for the combination of $\mathrm{S}_{0}$ and $\mathrm{T} 2 *$.

Conclusions: Results show that MRI of the lung has a similar diagnostic power compared with that of micro-CT regarding the detection of I/R injury after experimental lung transplantation. Both modalities provide complementary information in the assessment of dense and slight infiltration in the early phase after lung transplantation. Therefore, UTE MRI seems to be a promising addition to computed tomographic imaging in the assessment of $I / R$ injury after lung transplantation.

Received for publication May 10, 2013; and accepted for publication, after revision, July 11, 2013.

From the *Institute of Diagnostic and Interventional Radiology, and $\dagger$ Division of Thoracic Surgery, University Hospital Zurich; and †Institute for Biomedical Engineering, University and Swiss Federal Institute of Technology Zurich, Zurich, Switzerland.

Conflict of interest and sources of funding: Supported by the Swiss National Fund grant 310030_144075/1 and R'Equipe grant 3106030_139258/1.

The authors report no conflicts of interest.

Reprints: Moritz C. Wurnig, MD, MSc, Institute of Diagnostic and Interventional Radiology, University Hospital Zurich, Raemistr. 100, 8091 Zurich, Switzerland.

E-mail: moritz.wurnig@usz.ch

Copyright $(2013$ by Lippincott Williams \& Wilkins

ISSN: 0020-9996/14/4901-0023
Key Words: lung transplantation, ischemia-reperfusion injury, ultrashort echo-time MRI, microcomputed tomography

(Invest Radiol 2014;49: 23-28)

schemia-reperfusion (I/R) injury affects up to $30 \%$ of all patients undergoing lung transplantation. ${ }^{1}$ It constitutes the most common cause of death within the first 30 days after lung transplantation ${ }^{2}$ and affects the occurrence of bronchiolitis obliterans, ${ }^{3}$ which is one of the most important late complications. I/R injury is characterized by nonspecific alveolar damage, lung edema, and hypoxemia. Clinically, the symptoms vary from mild hypoxemia associated with few infiltrates to a picture similar to a full-blown acute respiratory distress syndrome requiring positive pressure ventilation, pharmacologic therapy, and, occasionally, extracorporeal membrane oxygenation.

Today, high-resolution computed tomography (CT) is the reference standard for anatomic imaging of the lung, ${ }^{4}$ offering the possibility to acquire diagnostic data even without suspended respiration. ${ }^{5}$ However, with the increase in resolution and signalto-noise-ratio, the amount of radiation applied also increases. ${ }^{6}$ Magnetic resonance imaging (MRI) offers an alternative without the need for ionizing irradiation, but because of the physical properties of lung tissue (low spin density and magnetic field inhomogeneities due to tissue-air interfaces), conventional MRI sequences with echo times (TEs) of 2 to 10 milliseconds cannot depict lung parenchyma at high field strengths. For that purpose, MRI sequences with ultrashort echo time (UTE) (100 $\mu \mathrm{s}$ and lower) and radial k-space sampling have been proposed. ${ }^{7-9}$ With these sequences, the assessment of tissue characteristics such as T1 time and $\mathrm{T} 2 *$ time $^{10,11}$ or attenuation coefficients is possible as well. ${ }^{12}$ Recently, it has been shown that in vivo MRI of $I / R$ injury and characterization of the tissue relaxation properties are feasible in a mouse model. ${ }^{13}$ Studying mouse models offers various advantages, for instance, the possibility to specifically investigate the pathological processes leading to $\mathrm{I} / \mathrm{R}$ injury ${ }^{14}$ or the outcome of experimental treatments. ${ }^{15}$ To this end, a noninvasive assessment via imaging would be advantageous and might help to improve $\mathrm{I} / \mathrm{R}$ injury evaluation after lung transplantations.

Although there are studies that showed the potential of UTE sequences in MRI of the lung,,$^{7-9,13,16-18}$ to our knowledge, only 1 study compared MRI of the lung (by using single-point imaging) to CT as the reference standard but assessed a mouse model with lower-thannormal lung density. ${ }^{19}$ The purpose of the present study was to compare the relaxation parameters obtained from UTE MRI data with the density parameters obtained from micro-CT as the reference standard for detection of I/R injury in a lung transplantation mouse model.

\section{MATERIALS AND METHODS}

\section{Animals}

Twelve C57BL/6 (H-2Kb) mice were used in this study: 6 of them served as donor; 6 , as recipients. The identical genome between 
donor and recipient allowed studying $\mathrm{I} / \mathrm{R}$ injury exclusively. The animals were approved by the institutional animal care committee (license number 177/2010) and received adequate care in accordance with The Principles of Laboratory Animal Care (promulgated in 1985 and most recently revised in 1996). ${ }^{20}$ No special preparation of the animals for the experiment was needed, except the adaptation and housing conditions.

\section{Transplantation}

The transplantation procedure was performed as previously reported by Jungraithmayr et al. ${ }^{13}$ In short, anesthetized and ventilated donor mice received laparotomy and sternotomy. After ligation of the trachea and preparation of the lung, the ascending aorta, and the root of the left pulmonary artery, the lungs were flushed with $2 \mathrm{~mL}$ of cooled $\left(4^{\circ} \mathrm{C}\right)$ storage solution. Ventilation was arrested at two-thirds of end-tidal inflation to ensure oxygen diffusion during the subsequent cold-ischemia time. The heart-lung block was then excised and stored in cooled $\left(4^{\circ} \mathrm{C}\right)$ preservation solution.

Graft preparation and orthotopic left lung transplantation were then performed using an operating microscope (Wild, Heerbrugg, Switzerland) at $\times 40$ magnification. After dissection of the left lung, the left pulmonary vein, the left pulmonary artery, and the left main bronchus were provided with a cuff made of a Teflon intravenous catheter (BD Insyte; Becton Dickinson, Madrid, Spain). After placement of a microvessel clip $(5.0 \times 9.0 \mathrm{~mm}$; closing force, $0.25 \mathrm{~N}$; FD562R; Braun-Aesculap, Tuttlingen, Germany) to avoid inflow, the graft was stored for 6 hours in the preservation solution at $4^{\circ} \mathrm{C}$ to induce $\mathrm{I} / \mathrm{R}$ injury.

The recipient mice received premedication analgesic $(0.1 \mathrm{mg} / \mathrm{kg}$ buprenorphine; Temgesic; Reckitt Benckiser, Slough, England) before the transplantation procedure. After assurance of adequate anesthesia, the mice were ventilated and a left-sided thoracotomy was performed within the fourth intercostal space. After retraction of the recipients' native lung, the left pulmonary vein, the left pulmonary artery, and the left main bronchus were separated, incised, and, via insertion of the respective graft cuffs, connected to the graft. The anastomoses were completed by fixating the cuffs with a provided 10-0 nylon ligature. Reperfusion was restored, and the lung was inflated with a brief application of positive inspiratory pressure of a maximum of $20 \mathrm{mbar}(2 \mathrm{kPa})$. After positioning of the transplanted lung into the recipient's thorax and excision of the native lung, the thoracic wall was closed in 3 layers with a 6-0 suture. The orotracheal tube was removed when spontaneous respiration resumed. After the transplantation procedure, all mice were allowed to recover in a warm, bedded cage and adequate analgesic medication was given.

\section{Micro-CT}

Micro-CT imaging was performed within 24 hours after the lung transplantation. All animals were anesthetized using isoflurane (Attane; Minrad I, Buffalo, NY), placed supine on the scanner bed, and kept warm using the provided heating mechanism of the physiology unit. All micro-CT imaging was performed on a high-resolution micro-CT (Skyscan 1176; Bruker-microCT, Kontich, Belgium). After an initial localizer scan, images were acquired using the following scan parameters: tube voltage, $50 \mathrm{kV}$; tube current, $500 \mathrm{~mA}$; exposure time, 80 milliseconds; rotation step, 0.7 degrees; covered angle, 360

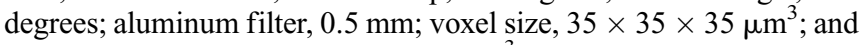
field of view (FOV), $20 \times 35 \times 35 \mathrm{~mm}^{3}$. To improve image quality, scanning was performed with prospective triggering using the supplied video monitoring tool and all images were acquired in end expiratory state. The duration of 1 scan was 15 to 20 minutes, depending on the breathing rate of the animal. The images were reconstructed with a modified Feldkamp algorithm, and the following parameters were used: beam hardening correction, 25\%; ring artifact reduction, 6 ; and Gaussian smoothing kernel with full width at half maximum of 4 voxels. The images were calibrated to Hounsfield units (HUs) via measurement of a phantom.

\section{Magnetic Resonance Imaging}

The animals were allowed to recover for 4 to 6 hours after the CT imaging before the MRI was performed. For the MRI, a Bruker 4.7-T PharmaScan 47/16 US with a gradient strength of $375 \mathrm{mT} / \mathrm{m}$, a slew rate of $3375 \mathrm{~T} / \mathrm{ms}$, and a linearly polarized proton wholebody mouse radiofrequency coil was used. The animal bed was equipped with a pad with continuous warm water supply to prevent cooling of the mouse. During the scan, the mice were anesthetized with isoflurane (Attane; Minrad I, Buffalo, NY). After gradient-echo localizers in 3 spatial directions, the imaging protocol consisted of a 3-dimensional T1-weighted spoiled gradient-echo sequence (repetition time [TR]/echo time [TE], 15 milliseconds/4.7 milliseconds; matrix, $192 \times 192 \times 32$; FOV, $40 \times 40 \times 45 \mathrm{~mm}^{3}$; and number of acquisitions, 3) and a 2-dimensional T2-weighted fast spin-echo sequence (TR/TE, 2500 milliseconds/11 milliseconds; effective TE, 33 milliseconds; echo train length, 8 ; matrix, $256 \times 256$; FOV, $40 \times$ $40 \mathrm{~mm}^{2}$; section thickness, $1 \mathrm{~mm}$; and number of acquisitions, 3 ). For $\mathrm{T} 2 *$ relaxation measurement, a 3 -dimensional radial UTE sequence was performed with 8 subsequent acquisitions with TEs of $50,75,100,500,1500,3000,4000$, and $5000 \mu \mathrm{s}$. The duration of the nonselective block-shaped excitation pulse was $20 \mu \mathrm{s}$, and TE was calculated as the time between pulse center and the start of the gradient ramp. Further protocol parameters of the UTE sequence were as follows: TR, 8 milliseconds; matrix, $128 \times 128 \times 128$; FOV, $45 \times 45 \times 45 \mathrm{~mm}^{3}$; spatial resolution, $350 \times 350 \times 350 \mathrm{\mu m}^{3}$; flip angle, 5.0 degrees; number of acquisitions, 1; and acquisition time, 6 minutes and 50 seconds per echo.

\section{Histologic Examination}

Immediately after the last imaging session, the animals were euthanized via application of carbon dioxide. The middle one-third of the pulmonary graft (approximately $30 \%$ of the whole-lung tissue) was recovered for histologic evaluation. The middle part of the lung graft was chosen because it representatively and best reflects all areas of the lung parenchyma, both large and small vessels and bronchi, the lung periphery, and the central parts of the lung. After formalin fixative was applied, the specimens were embedded in paraffin and $4-\mu \mathrm{m}$ cut sections were stained with hematoxylin-eosin.

\section{Data Analysis}

The micro-CT and magnetic resonance images were manually realigned. For each mouse, matching regions of interest (ROIs) were drawn correspondingly on 3 slices (apical/medial/basal) of the micro$\mathrm{CT}$ and magnetic resonance (MR) images. To avoid signal contribution from macroscopic vessels, only subpleural ROIs were used. To assess the image quality of the micro-CT and MR images, contrast-to-noise ratios (CNRs) were computed. Contrast-to-noise ratio was defined as follows:

$$
C N R=\frac{\left|S_{\text {transplanted }}-S_{\text {native }}\right|}{S D_{\text {background }}}
$$

with $S_{\text {transplanted }}$ and $S_{\text {native }}$ being the measured mean signal intensities within the ROIs of the respective side of the lungs and $S D_{\text {background }}$ being the standard deviation of the background signal. For MRI, CNR was computed on the basis of the UTE images acquired with a TE of $50 \mu \mathrm{s}$. In the micro-CT images, the mean HU density within the ROI was taken to measure image quality.

From the MRI UTE data, parametrical maps of the T2* time constant and the $S_{0}$ value, which is a value proportional to spin density, were calculated by pixelwise monoexponential fitting via scripts written in Matlab (The Mathworks, Natick, MA). All signal 

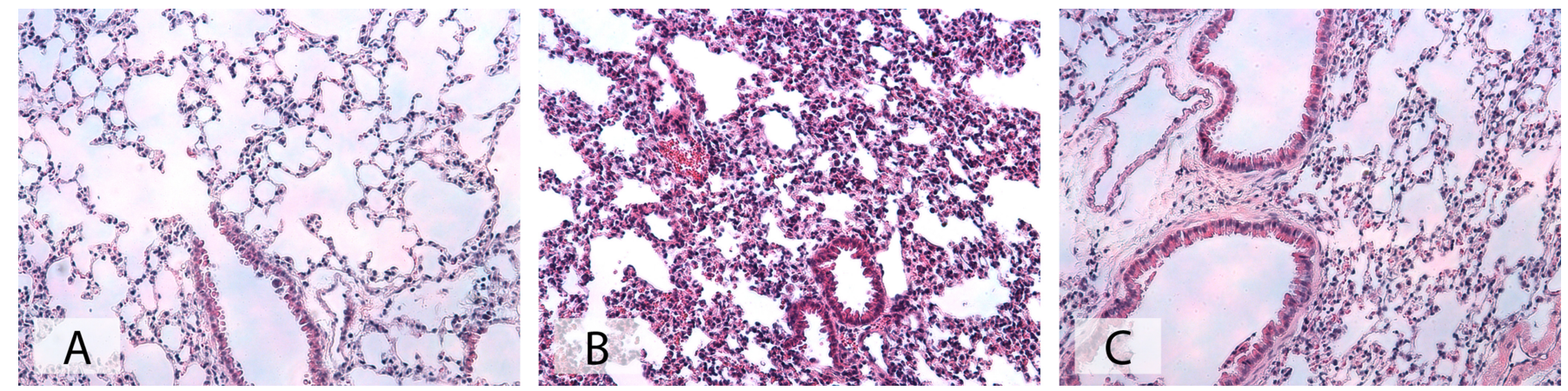

FIGURE 1. Hematoxylin-eosin-stained representative samples of the lungs show normal lung tissue in the non-transplanted lung (A) and various degrees of infiltration by immune cells as a correlate to I/R injury in the transplanted lung (B-C).

intensities of the MR images were previously corrected for different receiver gain values using the calibrated receiver gain function and for the automatic mapping of the data to the image gray value scale. The fitting routine used a nonlinear least-squares fit of the measured signal intensities to the following expression

$$
S(t)=S_{0} \times e^{-\mathrm{t} / \mathrm{T} 2^{*}}+N
$$

via an algorithm based on the interior-reflective Newton method. Fitting variables were $S_{0}$ and the T2* time constant. The noise term $N$ was not fitted but estimated as the standard deviation in an ROI selected in the image background outside the object avoiding regions masked due to the radial acquisition. For MRI tissue characterization, the mean $\mathrm{T} 2 *$ time and the mean $S_{0}$ value were measured via the ROI analysis.

\section{Statistics}

For statistical analysis, mean values and standard deviations were computed. All variables were tested for normal distribution with the Kolmogorov-Smirnov test. Depending on the result of the Kolmogorov-Smirnov test, a 2-sided unpaired Student $t$ test or a Mann-Whitney $U$ test was used to test for a statistically significant difference of variables between the ROIs within the transplanted lung and the ROIs within the normal lung. Accordingly, Pearson correlation coefficients or Spearman correlation coefficients were calculated. Receiver operating characteristic (ROC) curves and area under the curve (AUC) values were computed for the parameters $\mathrm{HU}$ density, $S_{0}$ value, and T2* value to evaluate their discriminative power between $\mathrm{I} / \mathrm{R}$ injury and normal lung tissue. To gain a single parameter combining the information of $S_{0}$ value and T2* value, the following logistic-regression model was fit:

$$
\operatorname{logit}(Y)=\beta_{0}+\beta_{1} \times S_{0}+\beta_{2} \times T 2^{*} .
$$

As dependent variable $Y$, the binary-coded status of the respective ROI was chosen ( 0 , non-transplanted side, no I/R injury; 1 , transplanted side, I/R injury). $S_{0}$ and $T 2 *$ were the respective measured values within the ROI, and $\beta_{0}, \beta_{1}$, and $\beta_{2}$ were the model regressors.

All $P$ values less than 0.05 were considered to be statistically significant. All statistical analyses were performed using commercially available software (Statistical Package for the Social Sciences 17.0; SPSS, Inc, Chicago, IL).

\section{RESULTS}

\section{Histologic Examination}

In the hematoxylin-eosin staining, areas with normal lung tissue without pathological findings and areas with slight reactive infiltration due to surgery were observed on the non-transplanted side of the lungs. In the transplanted lungs after the exposure to 6 hours of ischemia and the subsequent reperfusion at the time point of the implantation, the typical histologic picture of $I / R$ injury was found to be characterized by accumulation and infiltration of innate immune cells and alveolar edema. A representative picture of $I / R$ ischemic damage of the transplanted lung is provided in Figure 1.

\section{In Vivo Imaging}

The results of both MRI UTE and micro-CT showed an excellent depiction of the normal lung parenchyma and the pulmonary infiltration due to $\mathrm{I} / \mathrm{R}$ injury (Fig. 2). The ROI analysis results showed higher HU density in micro-CT (mean [SD] HU density, 29 [35]), higher MRI spin density (mean [SD] $S_{0}$ value, 2310 [300]), and higher $\mathrm{T}^{2} *$ time (mean [SD] T2*, 4550 [3230] $\mu \mathrm{s}$ ) in the transplanted left lung than in the normal right lung (mean [SD] HU density, -416 [120]; mean [SD] $S_{0}$ value, 1655 [440]; mean [SD] T2*, $895[870] \mu \mathrm{s})$. All differences in the micro-CT and MRI parameters between the transplanted left lung and the normal right lung were statistically significant $(P<0.001$ for all comparisons).

The image analysis results revealed that MRI provided a higher CNR between the normal and transplanted lungs compared

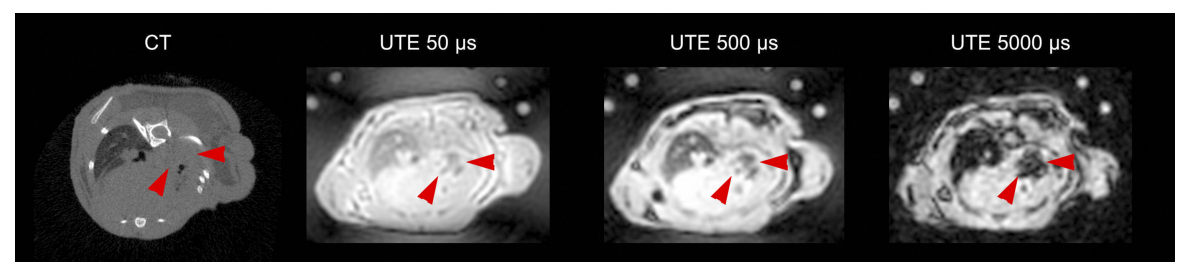

FIGURE 2. Typical depiction of lung tissue with and without I/R injury in micro-CT and UTE MRI. The results of both modalities show a high contrast between the normal lung parenchyma and the pulmonary infiltration due to I/R injury. Note that UTE MRI could detect air within the parenchyma of the transplanted lung (arrows), which was not possible with micro-CT. 

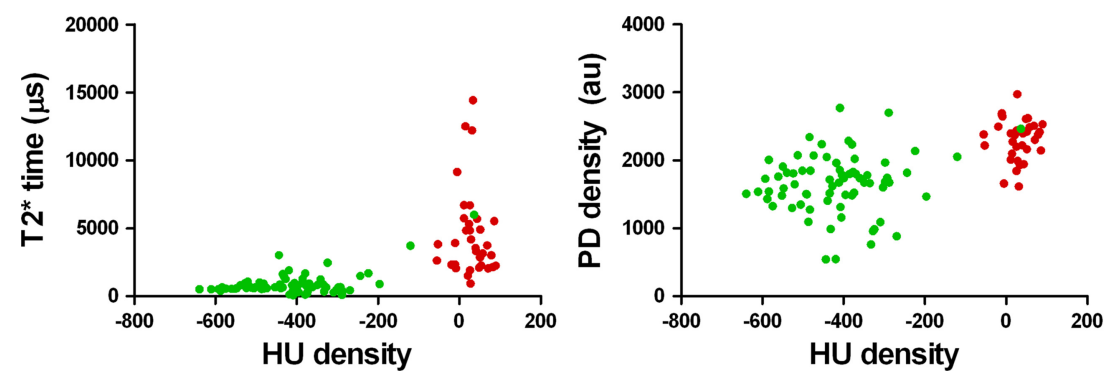

- left Lung (transplanted)

- right Lung (not transplanted)

FIGURE 3. Scatterplots comparing tissue characteristics measured with UTE MRI (T2* time and spin density) and micro-CT (HU density). Note that lungs with and without I/R injury can be excellently distinguished with HU density and T2* time values, although providing a different type of contrast.

with micro-CT (mean [SD] CNR MRI, 19.7 [8.0]; mean [SD] CNR micro-CT, 10.3 [2.5]; $P<0.001)$.

Results of the visual comparison of the micro-CT images and the $\mathrm{T} 2 *$ maps showed that slight infiltration in the non-transplanted lung could be better discriminated with micro-CT, whereas, in strong infiltration in the transplanted lung, a better contrast was provided by UTE MRI. This finding was confirmed in the correlation analysis, in which the areas with low T2* values showed high contrast of HU density and the areas with high HU density exhibited high contrast of T2* values (Fig. 3). On the other hand, little contrast was found in micro-CT for dense lung tissue. In dense lung areas with strong infiltration due to $\mathrm{I} / \mathrm{R}$ injury, the MRI measurements showed to be superior compared with those of micro-CT because only the UTE scans were able to demonstrate persisting ventilation of these alveolar lung areas. In micro-CT, only ventilation of the large airways could be seen preventing a clear differentiation between atelectasis and I/R injury (Fig. 4).

An advantage of micro-CT imaging was the depiction of the cuffs used for anastomosis of the pulmonary artery, pulmonary vein, and the main bronchus and, therefore, the possibility to evaluate the status of the bronchus cuff. Such an evaluation was possible in 6 of the 6 animals in micro-CT opposed to 0 of the 6 animals in MRI exhibiting signal loss artifacts because of spin dephasing at the place of the cuffs, thereby preventing an exact assessment of correct bronchus cuff placement.
The results of the correlation analysis showed a significant correlation between HU density and $S_{0}$ value $(\rho=0.60 ; P<0.001)$ as well as a significant correlation between HU density and T2* values $(\rho=0.67 ; P<0.001)$.

The results of the ROC curves and AUC analysis confirmed the excellent ability of micro-CT and MRI to distinguish between normal lung tissue and I/R injury (Fig. 5). The AUC value for $\mathrm{HU}$ density was 0.99 and amounted to 0.89 for $S_{0}$ value, 0.96 for T2* values, and 0.98 for the combination of $S_{0}$ values and T2* values obtained by logistic-regression model fitting.

\section{DISCUSSION}

In clinical radiology, high-resolution computed tomography remains the criterion standard for detection of acute pathologies after lung transplantation. In recent years, notable progress was achieved in the development of MR sequences and especially UTE sequences overcoming the obstacles of lung MRI. In this study, we investigated the potential of UTE MRI to detect I/R injury in a lung transplantation mouse model in comparison with a micro-CT reference standard. Results show that both modalities can excellently depict changes within the lung parenchyma associated with $I / R$ injury, which was also measureable with high CNR values for both methods. Furthermore, these findings were supported by the results of the ROC curves and the corresponding AUC values, which showed an almost equal ability to distinguish $\mathrm{I} / \mathrm{R}$ injury from normal

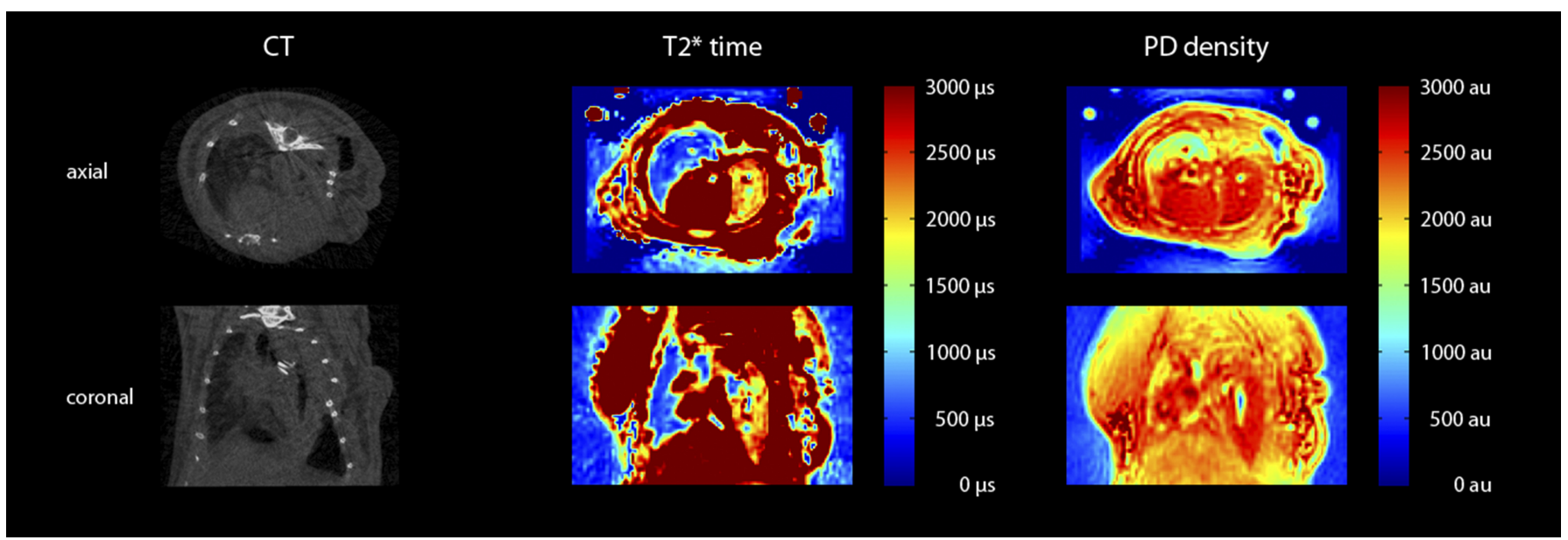

FIGURE 4. Comparison of T2* time and spin density maps with micro-CT images of a typical mouse with left lung transplantation and I/R injury. Only the T2* time maps were able to show ventilation of the transplanted lung. In contrast, only micro-CT could detect the cuffs used for anastomosis. 


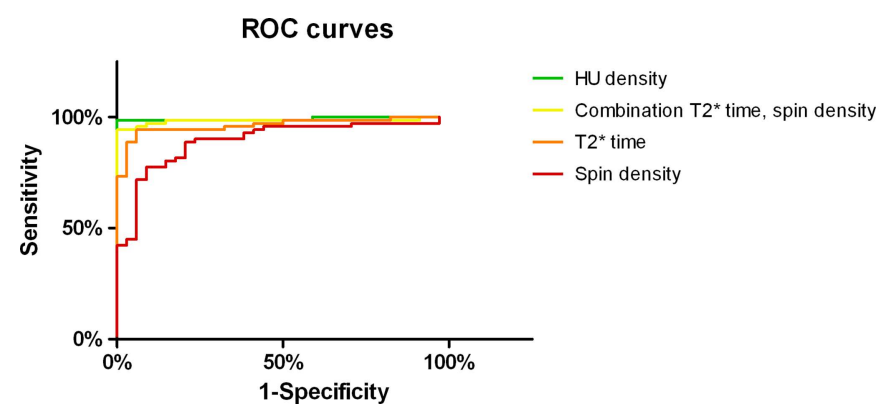

FIGURE 5. The ROC curves for the parameters measured with UTE MRI (T2* time, spin density) and micro-CT (HU density). The combination of both MRI parameters (computed via fitting of a logit model) has an almost identical diagnostic power, when compared with HU density.

lung parenchyma for both modalities. Also, both parameters that can be determined via UTE MRI, $S_{0}$ value and T2* value, were strongly correlated to HU density. Still, differences between UTE MRI and micro-CT could be observed: whereas micro-CT could better discriminate tissue with slight or no infiltration, UTE MRI could deliver better contrast in lung parenchyma with strong infiltration due to I/R injury. These complementary properties in the evaluation of dense and slight infiltration indicate that the combination of both modalities seems beneficial to provide comprehensive information on the graft status.

To the best of our knowledge, Olsson et $\mathrm{al}^{19}$ were the only ones so far to study the lungs of mice with micro-CT and MRI. The results of their study are in accordance with our findings. Because we examined a pathologic condition with a higher-than-normal lung tissue density, our study also extends the findings of Olsson et al, ${ }^{19}$ who studied an emphysema lung model with a lower-than-normal density. Furthermore, the study of Olsson et al ${ }^{19}$ did not investigate the correlation between the $\mathrm{T} 2 *$ time and HU density. This correlation is of interest because the $\mathrm{T} 2 *$ time is an intrinsic parameter of tissue properties that is often used to characterize tissue ${ }^{21-23}$ because it includes information about spin-spin interactions in contrast to spin density, which is naturally linked to tissue density. Also, T2* time is a tissue characteristic that can be measured quantitatively, whereas spin density needs a reference, for example, normal lung tissue in the same patient to be a meaningful parameter.

The findings of our study directly extend the study of Jungraithmayr et al, ${ }^{13}$ who could show that UTE MRI is feasible to detect and evaluate I/R injury in a mouse transplantation model because elevated $\mathrm{T} 2 *$ time and spin density correspond to histologic patterns of $I / R$ injury. Here, we could show that UTE MRI is comparable with micro-CT as reference standard regarding the general visualization of $\mathrm{I} / \mathrm{R}$ injury. Besides the lack of ionizing radiation, UTE MRI offers several potential benefits over micro-CT. First of all, UTE MRI can provide contrast where no other imaging technique could provide contrast so far, which might help to better characterize pathology in vivo. For instance, ventilation of the graft parenchyma could only be detected in MRI, whereas in micro-CT, the differentiation between $\mathrm{I} / \mathrm{R}$ injury and atelectasis was not possible because, virtually, no contrast was detectable within the transplanted lung. Furthermore, UTE MRI might offer the possibility to apply other helpful contrasts; for example, T1-weighted contrast might be suitable to detect bleedings after transplantation. Technically, such a contrast could be achieved via variation of the used flip angle. One disadvantage of UTE MRI, when compared with micro-CT, is its incapability to depict the cuffs used for anastomosis; therefore, the status of the bronchus cuff could only be assessed indirectly from the lung tissue. Increasing the resolution of UTE MRI might help to overcome this limitation in the future.

In the literature, a varying number of time points (from 2 up to 14 ) is used for fitting of the T2* decay. ${ }^{7-9,13,19}$ Although more time points are desirable for a more accurate fit, restrictions concerning scanner time and anesthesia time usually apply. In previous studies ${ }^{24}$ using lower field strengths, the use of multiecho spoiled gradient-echo sequences allowed the gathering of multiple echoes per repetition, which was not possible with the UTE sequence we used. To keep the stress for the animals used as low as possible, we decided to acquire 8 measurements with different echo times, resulting in good fits of the $\mathrm{T}^{*}$ decay while keeping the scan time reasonable. Another issue when not using a single multiecho sequence for quantitative measurements of the $\mathrm{T} 2 *$ time is the need to consider the correction for different receiver gain values and mappings, which was done in this study.

Regarding the computation of CNR values of MRI images, we chose an approach similar to that of Jungraithmayr et $\mathrm{al}^{13}$ and computed CNR on the basis of the signal intensities of the UTE sequence with the shortest TE. Of course, it would be desirable to compute CNR values on the basis of T2* and $S_{0}$ values. The reason for this approach is that, on the one hand, no exponential decay of MR signal intensities can be observed with UTE sequences in the ROIs containing air only; therefore, a meaningful fit, which allows the computation of T2*- and $S_{0}$ value-specific noise, was not possible. On the other hand, signal intensity of UTE sequences with very short TE should be a good substitute for spin density of the tissue.

Our study had limitations. Because micro-CT and MRI did not happen within the same session and placement and position of the mice within the respective scanner bed were not the same, only manual realignment was possible. Because the order of the 2 scans was not randomized, an influence of the time gap between the scans is thinkable. Nevertheless, it seems unlikely that this was the cause for the differences in the depiction of ventilated lung areas (appearing ventilated in MRI vs appearing not ventilated in CT) because we observed this pattern at different time points after the transplantation. Another limitation might be that no respiratory triggering was applied in MRI. The problem with respiratory triggering in UTE sequences is that an acquisition with 1 excitation per breathing cycle would lead to a significant increase in scan duration because of the increase in TR from the range of milliseconds to the range of seconds. A more feasible approach would be respiratory gating, which was just recently reported for a UTE protocol. ${ }^{18}$ Also, a limitation is the distribution of observed HU densities within the lungs, which was most probably caused by different pathomechanisms. Whereas an ischemia time of 6 hours with a following reperfusion resulted in heavy infiltrations correspondent to $\mathrm{I} / \mathrm{R}$ injury, the non-transplanted lung maximally showed slight infiltrations because of manipulation during the surgery. Although it would have been preferred, from a technical point of view, to include animals with less ischemia time and, therefore, slighter infiltrations, this was not considered to keep the suffering of the animals low.

We conclude that UTE MRI can excellently depict changes in the lungs due to $\mathrm{I} / \mathrm{R}$ injury and might serve as a complementary method to CT in the complete evaluation of transplanted lungs because of its different characteristic of contrast in areas with dense and slight infiltration.

\section{REFERENCES}

1. Lee JC, Christie JD, Keshavjee S. Primary graft dysfunction: definition, risk factors, short- and long-term outcomes. Semin Respir Crit Care Med. 2010;31: 161-171.

2. Christie JD, Edwards LB, Kucheryavaya AY, et al. The Registry of the International Society for Heart and Lung Transplantation: 29th adult lung and heartlung transplant report-2012. J Heart Lung Transplant. 2012;31:1073-1086. 
3. Daud SA, Yusen RD, Meyers BF, et al. Impact of immediate primary lung allograft dysfunction on bronchiolitis obliterans syndrome. Am J Respir Crit Care Med. 2007;175:507-513.

4. Ng YL, Paul N, Patsios D, et al. Imaging of lung transplantation: review. AJR Am J Roentgenol. 2009;192:S1-S13.

5. Baumueller S, Alkadhi H, Stolzmann P, et al. Computed tomography of the lung in the high-pitch mode: is breath holding still required? Invest Radiol. 2011;46:240-245.

6. Maher MM, Kalra MK, Toth TL, et al. Application of rational practice and technical advances for optimizing radiation dose for chest CT. $J$ Thorac Imaging. 2004;19:16-23.

7. Takahashi M, Togao O, Obara M, et al. Ultra-short echo time (UTE) MR imaging of the lung: comparison between normal and emphysematous lungs in mutant mice. J Magn Reson Imaging. 2010;32:326-333.

8. Togao O, Ohno Y, Dimitrov I, et al. Ventilation/perfusion imaging of the lung using ultra-short echo time (UTE) MRI in an animal model of pulmonary embolism. J Magn Reson Imaging. 2011;34:539-546.

9. Togao O, Tsuji R, Ohno Y, et al. Ultrashort echo time (UTE) MRI of the lung: assessment of tissue density in the lung parenchyma. Magn Reson Med. 2010; 64:1491-1498.

10. Springer F, Steidle G, Martirosian P, et al. Rapid assessment of longitudinal relaxation time in materials and tissues with extremely fast signal decay using UTE sequences and the variable flip angle method. Invest Radiol. 2011;46: 610-617.

11. Springer F, Martirosian P, Schwenzer NF, et al. Three-dimensional ultrashort echo time imaging of solid polymers on a 3-tesla whole-body MRI scanner. Invest Radiol. 2008;43:802-808.

12. Navalpakkam BK, Braun H, Kuwert T, et al. Magnetic resonance-based attenuation correction for PET/MR hybrid imaging using continuous valued attenuation maps. Invest Radiol. 2013;48:323-332.

13. Jungraithmayr W, Chuck N, Frauenfelder T, et al. MR imaging by using very short echo-time sequences after syngeneic lung transplantation in mice. Radiology. 2012;265:753-761.
14. Geudens N, Vanaudenaerde BM, Neyrinck AP, et al. Impact of warm ischemia on different leukocytes in bronchoalveolar lavage from mouse lung: possible new targets to condition the pulmonary graft from the non-heart-beating donor J Heart Lung Transplant. 2006;25:839-846.

15. Jungraithmayr W, De Meester I, Matheeussen V, et al. Inhibition of CD26/DPP IV attenuates ischemia/reperfusion injury in orthotopic mouse lung transplants: the pivotal role of vasoactive intestinal peptide. Peptides. 2010;31:585-591.

16. Conti G, Tambalo S, Villetti G, et al. Evaluation of lung inflammation induced by intratracheal administration of LPS in mice: comparison between MRI and histology. MAGMA. 2010;23:93-101.

17. Egger C, Cannet C, Gerard C, et al. Administration of bleomycin via the oropharyngeal aspiration route leads to sustained lung fibrosis in mice and rats as quantified by UTE-MRI and histology. PLoS One. 2013;8:e63432.

18. Johnson KM, Fain SB, Schiebler ML, et al. Optimized 3D ultrashort echo time pulmonary MRI. Magn Reson Med. 2012. doi: 10.1002/mrm.24570.

19. Olsson LE, Lindahl M, Onnervik PO, et al. Measurement of MR signal and T2* in lung to characterize a tight skin mouse model of emphysema using singlepoint imaging. J Magn Reson Imaging. 2007;25:488-494.

20. Bayne K. Revised Guide for the Care and Use of Laboratory Animals available. American Physiological Society. Physiologist. 1996;39:199, 208-211.

21. Rossi C, Sharma P, Pazahr S, et al. Blood oxygen level-dependent magnetic resonance imaging of the kidneys: influence of spatial resolution on the apparent R2* transverse relaxation rate of renal tissue. Invest Radiol. 2013;48 671-677.

22. Rossi M, Ruottinen H, Elovaara I, et al. Brain iron deposition and sequence characteristics in Parkinsonism: comparison of SWI, T(2)* maps, T(2)weighted-, and FLAIR-SPACE. Invest Radiol. 2010;45:795-802.

23. Welsch GH, Mamisch TC, Hughes T, et al. In vivo biochemical 7.0 tesla magnetic resonance: preliminary results of dGEMRIC, zonal T2, and T2* mapping of articular cartilage. Invest Radiol. 2008;43:619-626.

24. Boss A, Schaefer S, Martirosian P, et al. Magnetic resonance imaging of lung tissue: influence of body positioning, breathing and oxygen inhalation on signal decay using multi-echo gradient-echo sequences. Invest Radiol. 2008;43:433-438. 